\title{
Mandibular implant-retained overdenture with magnets in total edentulous patients (case report)
}

\author{
Edy Machmud, ${ }^{*}$ Sitti Arpa
}

Department of Prosthodontics, Faculty of Dentistry, Hasanuddin University, Makassar, Indonesia

${ }^{*}$ Correspondence to:

machmudedy@gmail.com

Received: 15 January 2019

Revised: 20 February 2019

Accepted: 10 March 2019

Available Online: 1 May 2019

\section{Abstract}

Objective: According to the manufacture, fewer than 1 in 10 capsule associated with overdentures on natural teeth separated from the denture base during an 8-year clinical trial; more interestingly, none experience loss of magnetic attraction.

Methods: Treatment for the restoration of oral function, including mastication, using mandibular implant-retained overdenture with magnets in total edentulous. Implant supported retained with magnetic attachment constitute an accurate and predictable treatment option and achieve a higher patients satisfaction.

Results: The surface of both magnet and attachment keeper were coated with titanium nitride (TiN). Self-curing resin was used for luting between magnets and denture base resin.

Conclusion: Magnetic attachments can be used to retain mandibular implant overdenture.

Keywords: Implant supported overdenture with magnets, Resorbed alveolar ridge, Total edentulous

Cite this Article:Machmud E, Arpa S. 2019. Mandibular implant-retained overdenture with magnets in total edentulous patients (case report). Journal of Case Reports in Dental Medicine. 1(1): 13-16. D0I: 10.20956/jcrdm.v1i1.91

\section{Introduction}

Continued bone loss in edentulous patients causes a great challenge in prosthetic rehabilitation by conventional denture. Retention has been the most problematic area for mandibular complete denture. Retention and stability are greatly compromised in conventional dentures, especially if mandibular ridge is severely resorbed. ${ }^{1}$ Implant-supported overdenture (ISO) as treatment in edentulous patients. ISO is a removable complete denture combined with implants designed to improve stability in the oral environment, provide greater retention, and support. Their hygiene is very easy and although they would need regular appointments for maintenance, they have a great acceptance by the patients. ${ }^{2-4}$

The advantage of implant retained overdenture are; ease for oral hygiene procedures; control of denture movement enhancing function and phonetics; the overdenture can be removed at bedtime to reduce effect of nocturnal parafunction; and increased masticatory efficiency, increased maximum myoelectric output, higher jaw-closing force, and less age-related bone loss after implantsupported prosthetic reconstructions than with a conventional complete denture. The 10 -year survival rates of implants to support mandibular overdenture tended to be slightly higher than survival rates of endosseous implants in the mandible used to support fixed full-arch bridges, fixed partial dentures or single crowns. ${ }^{1-3}$

ISO to be indicated in patients who cannot afford a fixed ISO or have anatomic limitations to implants or who have phonetic-aesthetic problems as loss of lip support, very long clinical crowns or wide interproximal spaces. ${ }^{3}$

Magnetic attachments consist of one magnet attached to the denture and another to the implant. They constitute a simple and comfortable system for the patient as magnet attraction guides the denture insertion. In addition, they are susceptible to corrosion by saliva, explaining why they are clinically less often used.

However, a new generation of rare-eart magnetic attachment could improve their properties and be clinically more often utilized. These new attachments may still be a useful treatment option for edentulous patient with weak muscle disease such as Parkinson's disease patient, because they not only keep the denture stable, but also need less force to insert and remove the denture. ${ }^{3}$

Magnets made from aluminium-nickle-cobalt (AINiCo) alloy have been used in dentistry for many years. Initially, the repellent force of like magnetic poles was harnessed from open-field AINiCo alloy embedded in the base of upper and lower denture, so that the repellent forces would keep dentures on the residual ridge. However, this approach achieved little popularity because the force was weak, and the direction of the force was just as likely to repel the denture out of the mouth. A more popular method was to attach a ferromagnetic metal keeper (generally made of stainless steel) to the tooth or implant for attraction by a magnet embedded in the nearby denture base; this arrangement is known as a magnet-keeper unit. ${ }^{5}$ 
Newer magnetic system have been made available for prosthodontics in the past couple of decades with the introduction of alloy of the rare eartelemets samarium ( $\mathrm{SmCo}$ ) and, more recently, neodymium $(\mathrm{NdFeB})$ in closed-field systems. Rare-earth alloy produce a stronger and more stable magnetic force than was previously available because they have high magnetization and high resistance to demagnetization. In closefield system, the magnetic field or flux is contained within the magnet-keeper unit and yields a much greater attractive force per unit size than is possible with open-field system. The newer closedfield magnets also have a higher attractive force per unit size when the keeper and the magnet are in contact, although this force diminishes rapidly when the magnet and keeper lost contact. ${ }^{5}$

In addition, a new system has been introduced to seal the metal capsule around a magnet and thus to protect it from corrosion in the mouth. According to the manufacture, fewer than 1 in 10 capsule associated with overdentures on natural teeth separated from the denture base during an 8-year clinical trial; more interestingly, none experience loss of magnetic attraction. ${ }^{5}$
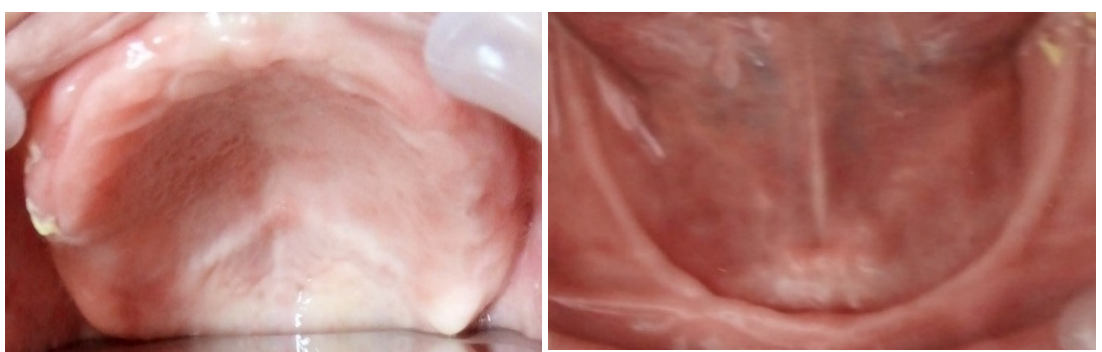

Figure 1 Intraoral view before implant treatment (the maxilla) and the mandible

\section{Case Report}

A 50-year-old women came to Dental Hospital Makassar Hasanuddin University wanted to fix a loose denture, which has been used for 9 years. Patients forget the last tooth extraction. Chief complaint of a mobile mandibular denture. The patient was completely edentulous and exhibited resorbed alveolar ridge figure 1 and figure 2 . She requested treatment for the restoration of oral function, including mastication, using mandibular implant-retained overdenture with magnets in total edentulous. After obtaining informed consent and confirming that the patient had no parafunction, two implant bodies of the mandible using single-stage implant surgery figure 3 . The implant right and left $12.0 \mathrm{~mm}$, diameter $4.0 \mathrm{~mm}$ figure 4 . The surface of both the magnet and the attachment keeper were coated with titanium nitride (TiN). Self-curing resin resin was used for luting between the magnets and the denture base resin figure 5 .

\section{Discussion}

In conventional dentures, there is an increase in bone loss and soft tissue abrasion due to horizontal movement of prosthesis under lateral loads. Mandibular jaw movement and action of muscle may lift the denture off the soft tissue causing difficulty in function and speech. To overcome these problems, implant retained overdenture is indicated. The placement of implants enhances the support, retention and stability of an overdenture. The continued bone loss after tooth and associated compromises in esthetics, function, and health, make all edentulous patients implant candidates, Hence, most completely edentulous patients should be informed of the necessity of dental implants to maintain bone volume, function, masticatory muscle activity, esthetics, and physiological health.

Two implants in mandible are normal minimal requirements. In a retrospective study, it was concluded that from both biologic and prosthodontic points of view, there were no differences in the performance of two implant supported dentures because the improvement in the retention, stability, and occlusal equilibration of the denture was related to increased size of the supporting implants and not to the number of implants. However, two implants supported overdenture require a minimum surgical intervention, are substantially less expensive to fabricate, easier to clean, readily accommodate esthetic and phonetic variable, provide better support for facial muscle, and offer higher patient satisfaction levels. ${ }^{6}$

This indicated that implant supported overdenture treatment should not be considered a second class treatment, rather in patients with mandibular denture retention problems and the ability to tolerated a removable denture, it is often the therapy of choice.

The implant placement in case was carried out with the expertise of the dentist and care was taken that there was least hinderance in making of prosthesis. The magnetic field system used is a closed field sistem that can reduce the external magnetic field. The magnet is mounted only on a denture base facing the abutment implant, made of ferromagnetic material. In a closed field system, the external flow of the magnetic field is removed by placing the magnetic component in a circuit and the two magnetic poles are placed in abutment implant. With abutment implant functioning to channel magnetic force lines, the spread of magnetic field to adjacent tissue can be limited and make the 

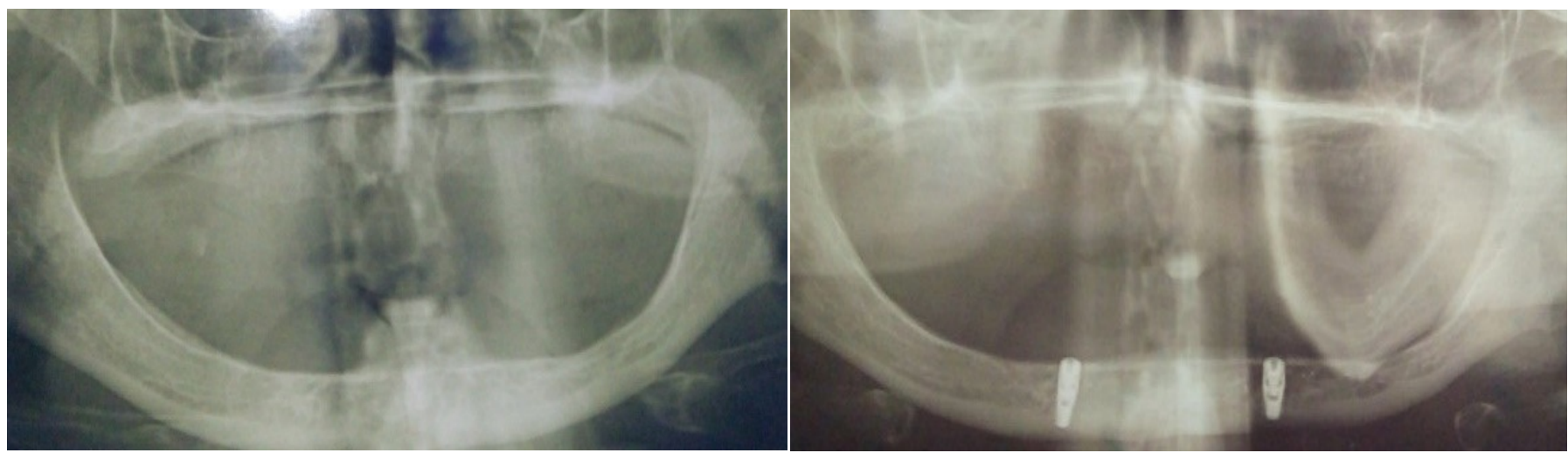

\section{Figure 2 Findings on a panoramic radiograph obtained before implant treatment}

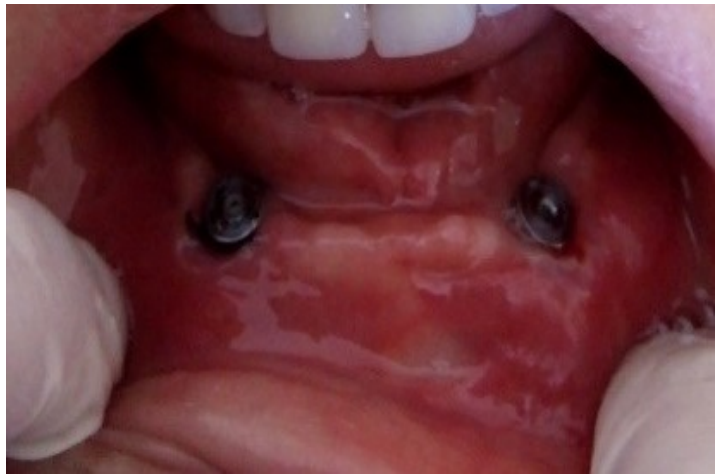

Figure 3 Two magnetic attachment supported by two implants

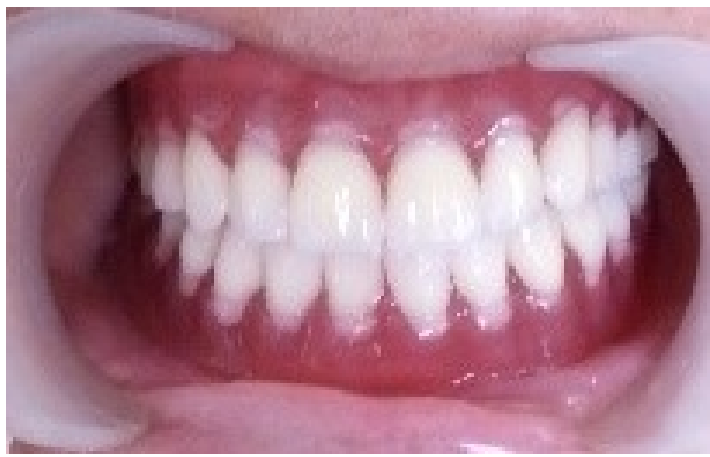

Figure 4 Conventional complete denture on the maxilla and mandible implant-retained overdenture with magnets

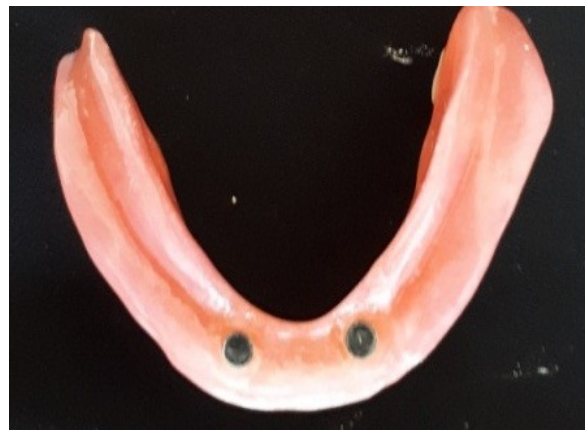

Figure 5 Two magnets bonded to the mandible overdenture attached more efficient to obtain retention and stability of denture teeth. ${ }^{7-9}$

\section{Conclusion}

Magnetic attachments can be used to retain mandibular implant overdenture. patient satisfaction over the first year was excellent, especially for patients who had been less than satisfied with mechanical attachment. This new generation of magnetic attachment can be applied in a straightforward manner and offers the potential for long-term durability.

\section{Aknowledgment}

None.

\section{Conflict of Interest}

The authors report no conflict of interest.

\section{References}

1. Warreth A, Alkadhimi, Sultan A, et al. Mandibular implantsupported overdenture: attachment system, and number and location of implants-part 1. J Ir Dent Assoc 2015;61: 93-7.

2. Juan F, Martines LA, Gustavo SA, et al. Rehabilitation with implant-supported overdenture in total edentulous patients: A review. J Clin Exp Dent 2013;5: e267-e272.

3. Kim HY, Lee JY, Shin SW, et al. Attachment system for mandibular implant overdenture: a systematic review. J Adv Prosthodont 2012;4: 197-203.

4. Taira Y, Sakihara M, Kamada, et al. Implantsupported overdenture retained with magnetic attachments. Asia Pac J Dent 2018;18: 15-20.

5. Ceruti P, Bryant SR, Lee JH. Magnet-retained implantsupported overdenture: review and 1-year clinical report. J Can Dent Assoc 2010;76: a52.

6. Vercruyssen M, Quirynen M. Long-term retrospective evaluation (implant and patient-centered outcome) of the two implant-supported overdenture in mandible. Part 2: marginal bone loss. Clin Oral Implant Res 2010;21: 466-72. 
7. Meenakshi A, Fatima N, Bharty V, et al. Magnet in prosthodontics. Int J Oral Health Med Res 2015;2: 81-4.

8. Shankar YR, Kumar TS, Gautam NS, et al. Magnet supported overdenture a case report. J Res Adv Dent 2014;3: 7-15.
9. Habar ID, Ardiansyah M, Surya ES, et al. Prosthodontic rehabilitation after traumatic anterior teeth and anodontia of mandibular right second premolar: a case report. J Dentomaxillofac Sci 2018;3: 129-131.

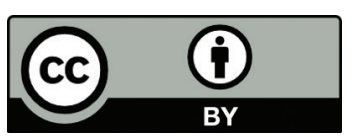

This work is licensed under a Creative Commons Attribution 